\title{
BOBI Y LA UTOPÍA DEL CINTURÓN VERDE
}

\author{
GLORIA FAVI PROFESORA, UNIVERSIDAD DEL PACÍFICO, CHILE.
}

La travesía delirante de Bobi desdibuja los límites de la ciudad segregada. Su búsqueda de un espacio utópico para su monstruosidad conduce a la ciudad imaginada, producción espacial del Estado de Chile para el hombre nuevo y una sociedad transformada.

PALABRAS Clave: Literatura - CHILE, PATAS DE PERRO, DROGUETT, SEGREGACIÓN URBANA, PSICO-GEOGRAFÍA, DERIVA.

\section{PATAS DE PERRO EN LA CIUDAD} DE SANTIAGO DE CHILE EN 1960

A través de la presencia transparente de un adolescente representado en la novela Patas de perro de Carlos Droguett ${ }^{1}$, intentaremos descifrar algunos signos cotidianos de la marginalidad social escenificados en un Santiago residencial y somnoliento,

Se trata del escrito publicado por primera $\mathbf{1}$ vez en Santiago de Chile por la editorial Zig-Zag en 1965.

Todas las citas del texto Patas de perro 2 corresponden a la primera edición a cargo de Pehuén Editores de septiembre de 1998. Colección Premios Nacionales de Literatura.

Desde la promulgación de la Ley de 3 Habitaciones Obreras de 1906 y la creación del Consejo Superior de Habitación Obrera, el problema de la vivienda popular no fue solo para la beneficencia que ejercía la Iglesia Católica y los católicos del Partido Conservador. El Estado chileno, en la mitad del siglo xx, fue actor participante en el proceso que culminó en 1953 cuando se dictó el Decreto con Fuerza de Ley No 224 y que dio origen a la Ley General de Construcciones y Urbanización para reglamentar el desarrollo científico del crecimiento urbano, inspirado en atractivos ejercicios urbanísticos foráneos, especialmente el Greater London Plan de Patrick Abercrombie (1944) y la utópica Brasilia, ciudad creada por Oscar Niemeyer (1957). Así nació el Plan Intercomunal para Santiago en 1960, en sustitución del antiguo plan Brunner-Humeres.

Según Armando de Ramón (200o), el 4 plan Brunner-Humeres patrocinaba el mantenimiento de la segregación espacial por estratos sociales. En la citada planificación, "calles y avenidas de residencias de lujo o de chalés con jardines al lado o hacia la calle" no debían situarse cerca de viviendas modestas porque afectaba su plusvalía. El escepticismo del urbanista Brunner radicaba en la supuesta "ignorancia del pequeño poblador" quien frente al fracaso de la ley de "habitación barata" y por falta de recursos, terminaba edificando "un rancho con cajones de automóviles, desechos de madera y latas viejas".

En el texto La semiósfera (1966), Iuri 5 Lotman demostró que la semiótica es una disciplina capaz de afrontar el estudio de la compleja vida social y de las relaciones que se establecen entre el mundo y el hombre. Lo más característico en el plano de la semiótica textual, es su tesis global sobre el funcionamiento del texto literario como signo cultural. Nuestra investigación está centrada en los signos culturales textuales que representan-en la literatura-

una particular producción y recepción enunciativa para comprender e integrar la comprensión del mundo real. espacio virtual que ha unificado su negativa violenta para albergar a Bobi, el deforme, cuya experiencia del paisaje de la ciudad de Santiago en 1960 ha condicionado nuestra percepción sobre la lenta y sostenida degradación urbana en el siglo XXI.

Pero ¿cuál es la historia de la ciudad que la memoria y los pasos entrecortados de Bobi conservan mientras nos desplazamos a través de los charcos, el barro y la sangre del barrio Matadero en este diseño vicario de fealdad y dolor?

\section{[...] miraba la sangre aguada chorreando por los lava-} bos, me quedaba mirando la cabeza desollada de un caballo y luego, luego estaban lloviendo los trozos de carne a mis pies, ellos me dejaban cogerla, ellos no me exigían sino un trocito y comerlo ahí, no me pedían más, esa era mi contribución. (Droguett, 1998, p. 94).²

La articulación de ese lenguaje construye la gestualidad del mundo imaginario que envuelve la monstruosidad de Bobi, mientras en esa ruptura siempre vigilada por la lógica y la historia legible, nuestra voz intenta cavar en las derruidas murallas citadinas para superar el olvido de la ciudad bárbara. Pero ¿cuáles son los códigos apropiados para (des)construir los espacios simbólicos y los sueños colectivos depositados en la memoria histórica de Santiago de Chile, en el ambular atormentado de Bobi y sobre una ciudad sin brazos, cuya exclusión de lo grotesco y deforme estaba organizada y demarcada en límites territoriales específicos?3

Bobi era oriundo del barrio Matadero-Franklin, periferia urbana de violencia y muerte que deslindaba en las aguas pestilentes del Zanjón de la Aguada, donde conventillos y ranchos pregonaban la inconsistencia de una programación estatal para construir viviendas populares. "Un barrio netamente obrero debe ser mantenido y limitado como tal"4 nos confirma el mundo real del plan Brunner-Humeres en 1939.

De esta forma, con los pasos de Bobi y Carlos nos proponemos reconstruir el espacio del discurso subalterno de los habitantes de Santiago de Chile en la mitad del siglo xx, ejercicio literario que nos permitirá resignificar, en tiempos diversos, un pasado y
The delirious journey of Bobi, the freak, blurs the boundaries of a segregated city. His search for a utopian space to contain its monstrosity leads to the imagined city, spatial production of the Chilean State for the new man and a transformed society.

KEYWORDS: LITERATURE - CHILE, PATAS DE PERRO, DROGUETT, URBAN SEGREGATION, PSYCHO GEOGRAPHY, DÉRIVE. English text in p. 23.

un presente al que le otorgaremos, mediante el giro discursivo, un nuevo sentido. El texto que he seleccionado para su estudio semióticos cumple una función activa en el mecanismo ideológico de la cultura en la mitad del siglo xx, en tanto es generador de un modelo de mundo en sus intentos para recuperar la conciencia activa del hombre social e histórico que se expresa en él.

La novela Patas de perro recupera parcialmente la memoria y representa los vestigios de la ciudad de Santiago, actualizados en los inicios del siglo xxI, cuya memoria y espacios públicos están siendo expoliados y expropiados por la concepción arquitectónica y paisajística (des)regulada que introduce el Estado moderno, y por la producción simbólica de Internet y la televisión, paradojas de una integración que propone individualismo y soledad con la navegación en los dispersos fragmentos migratorios de las autopistas virtuales.

Entonces observamos la incoherencia y el delirio de un narrador que en su relato intenta negar la supuesta irrealidad de la existencia de Bobi, un adolescente nacido en la iniquidad del barrio Matadero con unas hermosas patas de perro. Su decir nos revela en una red de pistas inconclusas, los fenómenos sociales asociados a la historia de Santiago de Chile entre 1950 y 1964. Esta situación contextual nos permite conjeturar sobre el hacinamiento de dos millones de habitantes en la capital, producto de las migraciones campesinas y mineras, más la desnutrición del $60 \%$ de la población de Santiago, considerada la ciudad más rica del país entre 1968 y 1969 (Collier y Sater, 1998).

En el relato, los pasos y miradas de Bobi sobre la ciudad y fachadas de las calles, descubren además a fantasmales sujetos anacrónicos que buscan un lugar donde vivir para esconder la tensión de sus miedos en esas barriadas disgregadas y carentes de toda compasión y compromiso emocional con las formas diferentes.

[...] pero comprendo que a otros, deformados espiritualmente, gente de comedores y de cocina, gente de platoy taza, tiene que dolerles, porque tú no eres un ser deforme sino todo lo contrario, porque tu forma es nueva y total $y$ ellos no lo son, son medio hombres, un cuarto de hombres... son alimañas, bestias domesticadas e infectas, voraces y calculadoras. (Droguett, 1998, p. 65)

El decir de Carlos, protector de Bobi y narrador de estos trágicos sucesos, se ha transformado en un acto de autonomía que reconstruye, en los distintos espacios de la ficción, los hechos de la violencia y 
FIG 1

Página 1 de la segunda edición de Patas de perro de Carlos Droguett.

Page 1 of the second edition of Patas de perro by Carlos Droguett.

Editorial Zig-Zag S. A., Santiago, 1966. Fuente/Source: Memoria Chilena,

Biblioteca Nacional de Chile.

Disponible en/Available at: www.

memoriachilena.cl segregación interiorizada en la ciudad real desde tiempos inmemoriales. Entonces conocemos desde qué exclusiones y contradicciones se construyen las identidades discursivas en el acto mismo de su participación como expresiones de una memoria espacial y conglomerados dialécticos de recuerdos que movilizan constantemente la lectura en los códigos secretos en nuestra ciudad.

Walter Benjamin nos revela en su "Crónica de Berlín" (1950):

Quien trate de acercarse a su propio pasado debe comportarse como un hombre que cava [...] Pues los estados de las cosas son solo almacenamientos, capas, que solo después de la más cuidadosa exploración, entregan lo que son los auténticos valores que se esconden en el interior de la tierra (p. 212).

Y los pasos de Bobi, generadores del recuerdo, evocan el mapa de su vida en el viejo barrio de vicio y miseria, mientras cavan un pasado donde objetos inquietantes yacen prisioneros:

"Sabía que me pegaría, le tenía verdadero terror a la hebilla de bronce de su cinturón, jamás se me olvidaba el olor del bronce metiéndose por los labios, una vez que me los partió" (Droguett, 1998, p. 170).
Su padre lo odiaba, pero pedía limosna para mostrarlo y hasta había comprado una linterna, para los sitios demasiado obscuros [...] (Droguett, 1998, p. 108).

El profesor Bonilla cogió el cuchillo de la mesa... Con paternal cuidado, levantó la mano y lo abofeteó y con la otra le lanzó una cuchillada...Bobi sintió la sangre que le corría por la mejilla" (Droguett, 1998, p. 163).

Walter Benjamin afirma: "El encuentro con el objeto libera el pasado que quedó atrapado en él"; fragmentos del pasado son entonces la hebilla de bronce, el cuchillo y la linterna, objetos cotidianos en el mundo real y que se han metamorfoseado, en el interior de este mundo violento, en productos de la excavación de posesiones intranquilizadoras que han adquirido su propia forma, peso y consistencia para organizar la sintaxis temporal que materializará las señas concretas y reales del exilio y destino trágico de Bobi. La música es el silbido del látigo que lo obliga a limosnear carne en los puestos sanguinolentos del matadero; el rayo de luz es la linterna que ilumina su deformidad mientras devora repulsivos trozos de carne; y el cuchillo es la destreza luminosa del profesor Bonilla mientras lo hiere en el baile de fin de curso. Estos objetos reales, en el mundo interno del relato, se constituyen como coordenadas geométricas

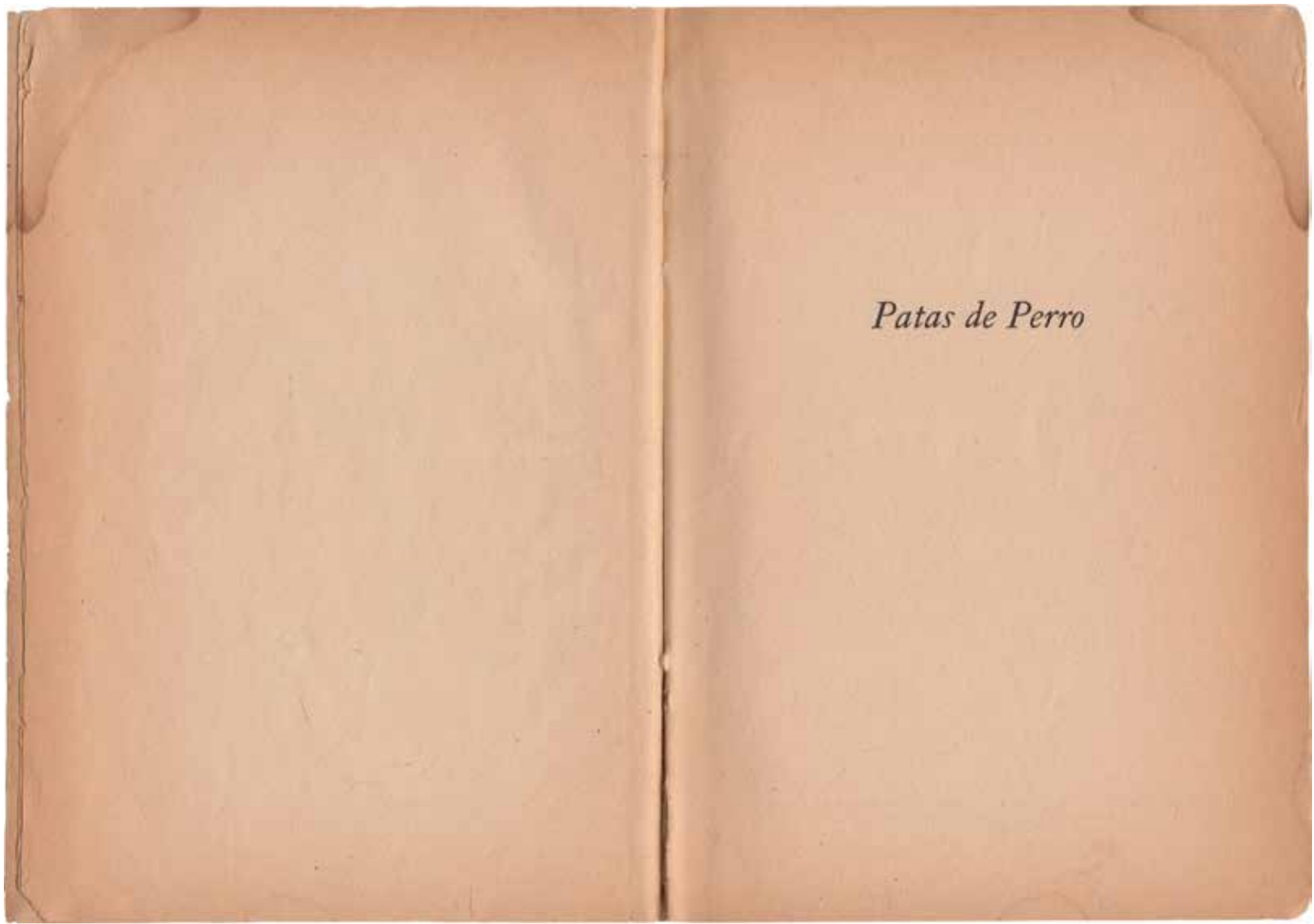


virtuales y temporales para demarcar los territorios desde donde es expulsado Bobi para generar, con sus pasos, el entramado de los sucesos sociales que darán forma a su historia.

De padres alcoholizados nacen hijos idiotas, monstruosos... otros los engendrany los botan al barrio, a la ciudad... es una insolencia tener al muchacho en una escuela que había sido abierta para recibir a seres humanos y no a engendros vomitados por la infraestructura del pais (Droguett, 1998, p. 81).

El decir del profesor Bonilla expulsa a Bobi, el monstruo perfecto, desde la ciudad letrada hacia los crueles embates de la jauría. Pero ¿desde qué institucionalidad habla? Creemos que en la construcción discursiva sobre la limitada ciudad de engendros, están contenidas, en calidad de símbolos, la violencia e ilegalidad de la exclusión territorial y social que ha condenado a la ciudad bárbara en todos los tiempos de nuestra historia.

Entonces necesitamos recuperar la historia de las palabras en la subversión sutil de un tiempo múltiple, para conocer en el siglo XXI, cómo se ha cristalizado el uso de las metáforas sobre la percepción del mundo de los excluidos en una determinada casta social.

De esta forma reiteramos la autonomía de Carlos, el narrador, cuyo delirio contradice las jerarquías y exclusiones desde la cual habla, porque las identidades discursivas que han generado su

En el texto Bicentenario: remodelación urbana. Utopías y anticipaciones para el hombre nuevo, su autor, Alfonso Raposo Moyano, reflexiona sobre el concepto remodelar y sobre la CORMU (Corporación del Mejoramiento Urbano, creada en 1965) como una de las identidades operativas que a partir de 1960 anticiparía los espacios para marcar el territorio que construiría el hombre nuevo. Se considera la producción del espacio urbano como un laboratorio social que emana desde la esfera pública creada por el aparato político administrativo del gobierno de la Democracia Cristiana.

El Plan Intercomunal de Desarrollo Urbano (1960) entre sus incisos proponía: I) Reforesta los terrenos de baja calidad agrícola cuyos bosques fueron destruidos por el consumo II) Implantar un sistema de "ciudades constelación", esto es, un conjunto de núcleos urbanos interrelacionados, de diferentes tamaños y luminosidades. III) Multiplicar los lugares y centros de esparcimiento en valles, lagos, lagunas y alta montaña. Chile. Ministerio de Obras Públicas.

La programación urbana y regional de Santiago de Chile que contiene el PRIs (Plan Intercomunal de Desarrollo Urbano 1960) y por otro lado, la propuesta de un plan MicroRegional para la cuenca de Santiago (1964), que incorpora la noción de regulación del paisaje natural en la periferia amenazada por la expansión urbana, intentaban potenciar los corredores ecológicos en el centro de Santiago. Se indica textualmente en uno de los incisos del pris: "Se regula el uso de las áreas suburbanas con el fin de defender e intensificar la producción agrícola, al mismo tiempo que se crea un cinturón verde entre las áreas urbanas suburbanas". Estos proyectos señalan la experencia relevante del Estado planificador chilen en sus intentos para racionalizar los servicios de urbanización, energía y transportes colectivos entre otros, se intentó mantener las forma urbanas incorporadas a la naturaleza en el diseño de los centros poblados.
6 hablar no están preestablecidas en la dimensión simbólica de una ideología. Estas identidades disidentes se construyen en los mismos actos discursivos que le permiten participar libremente en sus propios mundos de creencias y, nos proponemos reflexionar sobre las identidades en el texto, en tanto las consideramos como metáforas lingüísticas generadoras de la memoria espacial de la ciudad real en la mitad del siglo Xx. Estos conglomerados dialécticos de la memoria que se representan en el lenguaje de la literatura, nos permitirán invadir y descifrar parcialmente los focos disidentes de la ciudad bárbara en la iniquidad espacial de una aglomeración urbana que nos habla de la estructura económica y social de Chile en la década de 1950 a 1960.

"Cuando nació Bobi, su padre fue expulsado de la fábrica, su madre estuvo a las puertas de su muer8 te y tuvo que cambiarse de barrio, abrumado por la vergüenza y los insultos. En el almacén le cerraron la cuenta..." (Droguett, 1998, p. 93).

La institucionalidad barrial ha negado el vínculo con la originalidad del cuerpo de Bobi, sus iguales temen perder la conexión en el espacio colectivo y mental de la ciudad segregada, sin embargo, la rebeldía de Bobi en sus intentos para integrarse en la originalidad de "un espacio con ojos llenos de edad sumergida de sabiduría, que ya no lanzan miradas de odio, de furia, de soledad" (Droguett, 1998, p. 113) organizan el escenario de una experiencia urbana que nos acercará al discurso ideológico sobre la producción social de los espacios y lugares en la primera mitad del siglo $\mathrm{xx}$.
CARLOS Y BOBI, GENERADORES DE LA MEMORIA ESPACIAL EN EL SANTIAGO DE 1960

¿Soy yo un ser humano?... Tenía derecho a hacer esa pregunta... porque hasta ahora la vida se le habia presentado como una cualidad que no le correspondíay el mundo como un sitio al cual había llegado sin tener derecho a ello (Droguett, 1998, p. 111).

Esta interrogante confirma nuestros intentos por descifrar las identidades discursivas de Carlos y Bobi, en tanto consideramos su ambular y hablar disidente como dialécticas generadoras de la memoria espacial de la ciudad de Santiago en la mitad del siglo xx. Sabemos que los espacios arquitectónicos son territorios de subjetividades que crean significaciones reales para expresar la historia de una ciudad y su materialidad está condicionada por los marcos culturales de quienes la diseñan; así, los juicios y prejuicios perceptivos y receptivos que condicionan sus espacios y sociabilidades reflejan el devenir histórico de nuestras instituciones culturales, especialmente el escenario político en 1960, lugar utópico donde empiezan a inscribirse las primeras concepciones urbanísticas y arquitectónicas para integrar nuevos modelos de sociabilidades y movilizaciones populares cuyo eje central de transformaciones sociales es impulsado por el Estado chileno. ${ }^{6}$

La historia de Bobi es la experiencia urbana de un cuerpo disforme que la recorre para aventurarse a múltiples posibilidades de relaciones que siempre fracasan, y la escritura de Carlos es la cartografía de un cometido poético que ilustra la iniquidad excluyente del cuerpo colectivo de nuestra ciudad.

¿Cómo daremos forma al desequilibrio y desarmonía del cuerpo urbano y social en Santiago de Chile? se preguntaban los planificadores en la realidad espacial de la década del sesenta, mientras las coordenadas geométricas del desarraigo espacial en el interior del texto nos hablan:

\section{El profesor Bonilla agregó que ya era una insolencia} tener al muchacho en una escuela que habia sido abierta para recibir a seres humanos y no a engendros vomitados por la infraestructura del país [...] me contestó que dónde estaban las piernas del niño llamado Bobi [...] que por ahí le habian enviado un perro y que ésa no era escuela de perros ni circos ni zoológicos ni museo ni exposición" (Droguett, 1998, p. 84).

Los cambios en la producción del espacio auguraban transformaciones en la concepción paisajística para dar inicio a un nuevo orden social. De esta forma, la conceptualización de "espacios abiertos, áreas verdes, área suburbana" que preservan el patrimonio natural y cultural en Santiago de Chile, correspondería a la creación de una utopía espacial iniciada desde la esfera estatal con la promulgación en 1960 del Plan Intercomunal de Desarrollo Urbano (PRIS) ${ }^{7}$. Algunos urbanistas calificarían los proyectos y remodelaciones urbanas en 1960 como estrategias políticas para anticipar la aparición del hombre nuevo. ${ }^{8}$

Cuando Bobi definitivamente sale de su hogar en el barrio Matadero, abandonado por el certero alivio 
FIG 2

Introducción de Patas de perro de Carlos Droguett/Introduction to Patas de perro by Carlos Droguett.

Editorial Zig-Zag S. A., Santiago, 1966. Fuente/Source: Memoria Chilena, Biblioteca Nacional de Chile. Disponible en/Available at: www. memoriachilena.c de sus padres, y Carlos era abandonado por la mujer con quien se casaría, sus pasos y el itinerario de sus voces configuran la imagen y forma de una ciudad inseparable del tiempo y la memoria contenida en el desarrollo sustentable de los años sesenta y sus sistemas coordinados de espacios verdes destinados al esparcimiento de la población y saneamiento ambiental potenciado por corredores ecológicos que intentan unir lo urbano y lo rural (Pavez, 2009).

"Casa chica a matrimonio solo en barrio tranquilo, a una cuadra de la Gran Avenida. No se admiten perros" (Droguett, 1998, p. 72). La promesa del paraíso perdido contenida en el anuncio de un periódico es el tránsito frenético de Bobi y Carlos desde sus calles de pesadilla hacia el equilibrio y la belleza de la ciudad imaginada y al vasto depósito de una historia prometedora contenida en la salud de sus áreas y avenidas destinadas a cumplir, en el pasado, la utopía de una institucionalidad que regulaba la protección del paisaje natural amenazado por la expansión urbana de la metrópolis.

Buscar un lugar para vivir era además el residuo de los sueños colectivos de los pobladores hacinados sobre la iniquidad territorial de la ciudad real en $1960^{9}$, una ciudad cuya forma nueva y total sería la utopía del diseño y protección de áreas verdes, ríos, hondonadas y quebradas que puedan contener la monstruosidad perfecta de Bobi y el deseo de olvido de Carlos, en estos lugares diseñados para la perfección y toda la felicidad que les ha sido negada. Una terraza y un patio lleno de flores sería la construcción arquitectónica de un lugar para vivir, la utopía del cinturón verde que anuncia y anticipa una ciudad nueva en un pueblo silencioso, fuera de la hostilidad de la gran ciudad que excluye la monstruosidad perfecta de Bobi y que nos integraría en esta nueva producción del espacio urbano para postular a una sociabilidad diferente, hombres nuevos integrados en la plasticidad de formas abiertas, sentir, respirar el aire...

"Nos iríamos. Buscaríamos casa en el barrio del cerro. Encontramos una casa pequeñita con terraza y un patio lleno de flores, una cocinita minúscula, un baño limpio y sencillo..." (Droguett, 1998, p. 88).

Pero, las casas, las calles y las ciudades no son solo transeúntes y cosas, son también pensamientos, voluntades y participaciones y desde esos contextos, Bobi y Carlos son expulsados con la violencia imperativa de un lenguaje: "iLa casa es mía y yo tengo derecho para preguntarle en qué la usa usted y porqué la usa de este modo!" (Droguett, 1998, p. 70); "Mis clientes le notifican que debe desalojar la casa el 30 del mes. Atentamente, Gándara" (Droguett, 1998, p. 73). El fin de los sueños es la maldad infinita de la señorita Estefanía, en su boca desganada permanece la interpelación violenta del sentido común para excluir desde sus espacios normados, las formas nuevas y totales que representan las hermosas patas de perro de Bobi: "nos cambiaremos de casa ¿no es cierto? Si, Bobi, le dije, nos cambiaremos, buscaremos una casa agradable y pequeña" (Droguett, 1998, p. 81)

Entraremos a la casa, que veía luminosa ahora y como más grande... Puente Alto... sentíamos el aire seco lleno de ecos que bajaba asoleado de la montaña, al preguntarle yo si le gustaría irse a vivir a ese pueblo, provinciano, silencioso, apegado a la tierra [...] entre las altas montañas y junto a los ríos modestos y apacibles, él me quedó mirando lleno de alegría y me dijo que siempre le habia sorprendido que yo quisiera seguir en Santiago, cuando en la gran ciudad hostil no tenía nada que hacer [...] (Droguett, 1998, p. 111).

¿Puente Alto será el proyecto de la ciudad imaginada por Bobi y sus formas nuevas en el marco ficticio de una nueva legalidad espacial? ¿sería la utopía salvadora que lo rescataría del hacinamiento, hedor y pestilencia del barrio Matadero y de la trágica persecución institucional que encarnan su padre, la escuela y la policía? 
Bobi, le dije, en verdad la gente de este pueblo no tiene ojos o sus ojos son como los de los peces, pulidos largamente por el mar, ojos sin aristas que no duelen, ojos llenos de edad sumergida de sabiduría, que ya no lanzan miradas de odio, de furia, de soledad (Droguett, 1998, p. 113).

Y solo serán las miradas..."llenas de edad sumergidas y de sabiduría"... en estos espacios transparentes, quienes fijarán en un intercambio de subjetividades, la aceptación no formulada de la monstruosidad de Bobi. Así, Puente Alto será el último reducto de ensoñación que se ofrecerá a un Bobi moribundo y acechado por la institucionalidad que ejerce su prevención violenta contra los anormales.

\section{BOBI, LOS FAUNOS, LOS SÁTIROS $Y$ EL ESPACIO SAGRADO}

Desenterramos, desde el baile organizado en la escuela donde asiste Bobi, la luminosidad extraordinaria del mito dionisíaco ${ }^{10}$ en la dulce sensualidad que ejercen las formas perfectas de sus patas de perro en la comparación que lo une a sus disformes semejantes; los sátiros, faunos y centauros. En la negativa de este cortejo para aceptar el orden y horror que imponen los titanes, permanece latente la aceptación del divino éxtasis en el baile letárgico que actualiza y ejecuta Bobi para intentar la redención que lo uniría al mundo de los humanos. Desde esta perspectiva, nuestra lectura se convertirá en un ejercicio de imaginación que gestionará mapas efectivos para contener, desde el lenguaje y la memoria, espacios imposibles cuya transformación en reductos de ensoñación hace posible la utopía de las nuevas sociabilidades y los espacios paradisíacos que podría contenerla. ${ }^{11}$

Bailé, me dijo Bobi, bailé realmente con la Silvia, la Enriqueta, la Matilde, bailamos mucho, teníamos el calor en la cara, el sol en los labios, la música nos movía los pies, yo me cogía de sus cinturas, ellas se tornaban pálidas y pensativas, la Silvia me dijo que bailaba muy bien, que porqué nunca salía de noche, que en la noche brillan los bailes al final de la calle. Suspiró, se sonreía pálido, estaba feliz y olvidado, comenzó a silbar suavemente (Droguett, 1998, p. 159).

Pero ¿̇cuál es el sentido del mundo dionisíaco en los pasos y el baile que ejecuta Bobi para demarcar su espacio sagrado en la ciudad fragmentada? Hablamos entonces de su sabiduría e intuición en la búsqueda de la unidad perdida y la afirmación de la vida por sobre el dolor del rechazo y abandono más allá del optimismo de la superficialidad.

Friedrich Nietzsche afirma:

La vida es indestructiblemente poderosa y placentera, ese consuelo aparece con corpórea evidencia, como coro de sátiros, como coro de seres naturales, que por así decirlo, viven inextinguiblemente por detrás de toda civilización y que, a pesar del cambio de generacionesy de la historia de los pueblos, permanecen eternamente los mismos (Nietzsche, 1973, p. 77).
Sin embargo, comprendemos que no hay lugar para el baile orgiástico en la foresta y la fantasía de una senda mágica para establecer la unidad perdida se ha quebrado en las acciones estridentes del profesor Bonilla y la eficacia terrorífica de los titanes policiales y familiares; Bobi herido y humillado es obligado a abandonar el baile escolar luego de su triunfo y de los fervorosos aplausos de su público.

Este cuchillo... dijo el profesor y se sonreía ahora con miedo, con verdadero miedo, dará comienzo a la kermesse, no se asusten ustedes, sean valientes, miren lo valiente que es Bobiy se tornó hacia ély comenzó a aplaudirlo poniéndose el cuchillo entre los dientes y al momento estuvo gritando, se echó debajo de los peldaños y estaba chillando furioso, pero la gente no se levantaba y huía, la gente no se asustaba, estaba aplaudiendo [...] (Droguett, 1998, p. 163).

El público aplaudía fervorosamente y sus ninfas enceguecidas en el ritual del baile, integraban finalmente a Bobi, al hijo extraviado, en el sendero mágico de la unicidad. El olvido y la embriaguez confieren a Bobi el triunfo y exultación de sus formas perrunas, mientras las ménades de su cortejo participan en el vértigo de su transfiguración divina sobre el espacio mágico, el abismo que demarca su separación con "los otros". "Cuando sintió que lo llamaban y que lo tornaban a llamar y que sonaban algunos aplausos, se puso de pie, se colocó la máscara y caminó hacia afuera (...) (Droguett, 1998, p. 161).

Nos preguntamos ¿cuál es el sentido de la actuación violenta del profesor Bonilla frente al desborde del baile que ejecutan Bobi y sus compañeras? Retornamos entonces al mito báquico, a Eurípides, siglo v a.C. a algunas estrofas de Las Bacantes ${ }^{12}$ :

He oido de nuevos males en la ciudad que nuestras mujeres han dejado las casas con fingidas danzas para en los espesos montes entregarse al vértigo y al recién llegado Dios, ese Dionisio que no sé quién es, celebran con danzas acuden a gozar del concúbito de un hombre con el pretexto de ser Ménades rituales. Las encerraré en redes de hierro y las haré dejar enseguida ese criminal rito (versos 215-220).

Es el canto de Penteo, hijo de Equión, correcto funcionario, rey y guardián de las leyes en la ciudad de Tebas, representante de la norma, la medida y la razón opresora del Estado frente a la desmesura de la naturaleza (Eurípides, 2005).

Bobi miraba el patio lleno de lucesy de gentes y se sentía acobardado, admirado que cinco minutos antes hubiera estado él, él danzando gentilmente alrededor de una agradable perfumada vaporosa creatura, elevando sus manos con energía al ritmo del baile, adelantando su cara enmascarada y afiebrada hacia la boquita que le preguntaba (Droguett, 1998, p. 169).

Bonilla, oscuro pedagogo de ropas gastadas, atemorizado frente a la seducción que ejercen las danzas jadeantes de pasión por el triunfo 
de la existencia y el exuberante sentimiento de transfiguración, cual émulo de Penteo, rey de Tebas, siente el deber ineludible de aniquilar la desmesura y oponer la razón:

El profesor le arrebató la máscara y la levantó para que la aplaudieran [...] levantó la mano y lo abofeteó y con la otra le lanzó una cuchillada y la máscara saltó como una tapa de resorte y Bobi sintió la sangre que corría por sus mejillas [...] Si se va la música, pensó, me van a matar, y miró el rostro del profesor que lo atisbaba y lo trataba de bestia de asesino de monstruo depravado [...] (Droguett, 1998, p. 165).

La máscara y la música marcan la transfiguración y sacrificio del héroe, su cuerpo torturado y encadenado yace en un sucio cuartel policial; la desmesura aplacada por la razón representa el fin de una senda unificadora a la integración con la naturaleza, esos leves intersticios del paisaje urbano que permitirían atisbar el soplo helado, los ríos, las hondonadas, las quebradas ${ }^{13}$. Bobi representaría la última esperanza de redención en un universo cercado por titanes policiales "tú eres el portador misterioso de una revolucionaria forma de ser humano, mucho más noble, más leal [...] si la Naturaleza ha cambiado en tu cuerpo, te ha traído como un emisario, como un espía muy especial" (Droguett, 1998, p. 248).

Luego, la huida del cuartel policial lleva a Bobi a caminar por el San Cristóbal, las orillas del río Mapocho y la Quinta Normal y en esas trayectorias es imposible el ocultamiento y la fuga porque la imagen y la forma de nuestra ciudad es también nuestro cuerpo inseparable del tiempo y la memoria "un bote con enamorados en medio del agua, el vendedor de barquillos adormilado o resignado, el fotógrafo ambulante, francamente enojado y bárbaro".

Son nuestras visiones diluidas, descoloradas cual viejas tarjetas postales convertidas en nuestro cometido poético para recuperar en el dolor de Bobi, el tiempo, el recuerdo y las imágenes de nuestra ciudad destruida.

Entonces la aventura de Bobi no solo ha sido un asunto de cuerpos, un cuerpo solitario que sale de sí mismo y se expone a los otros en la exultación de una particular belleza: su huida se convierte en la proyección premonitoria de una nueva ciudad señalada por la banalidad y el sin sentido: Nasón (43 a.C.) "¿Qué delirio, hijos de la serpiente, descendientes de Marte se ha apoderado de vuestras almas? - dice Penteo- ¿Tanto poder tienen el bronce chocando con el bronce, la flauta de cuerno curvada y los engaños de la magia, que a los no aterrorizó ni la espada ni la guerra ni la trompeta ni los batallones erizados de armas les pueden vencer unas voces de mujeres, la locura que promueve el vino, una multitud de seres obscenos y unos tamboriles huecos?” (p. 43)

Según el MINVU: "El uso del suelo $\mathbf{1 4}$

debe regirse por disposiciones flexibles definidas por los requerimientos del mercado". Por otra parte, "se definirán procedimientos y se eliminarán

restricciones de modo de permitir el crecimiento natural de las áreas urbanas siguiendo las tendencias del mercado".

\section{Miras un mundo delgado y transparente, árboles} enormes pero de papel, un agua sucia y falsa, demasiado bien imitada, un agua arrugada de papel, hombres, mujeres de papel, risas de papel, palabras de papel que se van volando con el humo de carbón que viene de la línea del tren, nunca estuve tan suprimido de la vida como esta tarde en la Quinta, pero me fue agradable porque a mi lado pasaban los perros [...] (Droguett, 1998, p. 201).

Junto a Bobi nos preguntamos ¿cuán suprimidos estaremos de la vida real en el actual simulacro de las nuevas sociabilidades electrificadas? Porque Puente
Alto era el fin de nuestro viaje donde "no te miran a ti como si tú fueras el engendro que son ellos" (Droguett, 1998, p. 82). Pero comprendemos que esa nostalgia fue una trampa y que en esos espacios sagrados ya no existe salvación posible, el presente ha sido una condena, la eterna lucidez sin esperanzas. Puente Alto, fue transformado en los inicios del siglo XXI en lugares de sociabilidades en conflicto, ajeno y adverso a los códigos interpretativos de las políticas urbanas que proponía la utopía de las áreas verdes en 1960. Utopía que finalizó en 1979 con la presión del sector inmobiliario quien impuso la liberación de los mercados de suelo ${ }^{14} \mathrm{y}$ eliminó la norma sobre límites urbanos. Esa situación que produjo una grave segregación residencial, especialmente en la comuna de Puente Alto -destinatario selecto para las políticas de erradicación y ubicación de campamentos y poblaciones marginales, junto con marcar las estadísticas más altas de criminalidad y hacinamiento- junto con la polémica que ha generado la medición de la Encuesta CASEN 2012 para la calificación de 629.861 habitantes entre los cuales calificarían cien mil personas en condiciones de pobreza.

Entonces el ingreso de Bobi a la jauría es nuestro consuelo; Friedrich Nietzsche nos confirma:

Ese consuelo aparece con corpórea evidencia, como coro de sátiros, como coro de seres naturales, que por así decirlo, viven inextinguiblemente por detrás de toda civilización y que, a pesar del cambio de generacionesy de la historia de los pueblos, permanecen eternamente los mismos" (Nietzsche, 1973, p. 77).

Rescatamos para Bobi y su eternidad, la simulación aparente de un objeto que emerge semioculto entre tantas y tantas palabras. La flauta y las notas melancólicas que ejecutan el concierto para clarinete de Mozart, la flauta distraídamente cercana a un receptor de radio, la flauta aparentemente olvidada junto a ropas arrugadas, la flauta ahora blandida triunfalmente por Bobi, cuando sus huellas marcan la tierra húmeda que lo llevará a la jauría.

La flauta no, la flauta no estaba, fui al patio, salí a la calle obscura, miré a la tierra y alli estaban sus huellas, me agaché a mirarlas... iOh Dios!, grité con furia mientras las lágrimas reventaban en mis ojos (Droguett, 1998, p. 31). ARQ
GLORIA FAVI | Profesora de Castellano, 1971; Magíster en Literatura, 1990 y (c) Doctora en Literatura, Universidad de Chile. Es Investigadora en el Departamento de Investigación y Desarrollo de la Universidad de Chile desde 2007 y a partir de 2011 forma parte del comité de redacción de la Revista indexada Cifra Nueva, editada por la Universidad de Los Andes en Trujillo, Venezuela. Escribió el libro Crónicas de la marginalidad, editado por Bravo \& Allende con el auspicio de la Corporación de Desarrollo de las Ciencias Sociales. Actualmente es directora de la Asociación Chilena de Semiótica, profesora de Literatura Chilena e Hispanoamericana en la Universidad del Pacífico y consultora externa de diumce (Departamento de Investigación y Desarrollo de la Universidad Metropolitana de Ciencias de la Educación). 


\section{REFERENCIAS BIBLIOGRÁFICAS}

BENJAMIN, Walter. "Crónicas de Berlín".

En: Escritos autobiográficos. Madrid,

Alianza, 1996, p. 188-242.

COLLIER, Simon y SATER, William. Historia de Chile (1808-1994). Madrid, Cambridge University Press, 1998.

DAUDI, León. Prontuario de la mitología griega. Madrid, Zeus, 1965.

DE RAMÓN, Armando. Historia de Chile. Santiago, Catalonia, 2003

DE RAMÓN, Armando. Santiago de Chile.

Santiago, Editorial Sudamericana, 2000.
DROGUETT, Carlos. Patas de perro.

Santiago, Pehuén, 1998.

EURÍPIDES. Las Bacantes. Madrid, Gredos, 2005

OTMAN, luri. La Semiósfera I. Madrid, Cátedra, 1996.

MONGIN Olivier. La condición urbana. La ciudad a la hora de la mundialización. Buenos Aires, Paidós, 2006. NIETZSCHE, Friedrich. El nacimiento de la tragedia. Alianza Editorial, Madrid, 1973. OVIDIO NASÓN, Publio. Las Metamorfosis. México, Editorial Porrúa, 1994.
PAVEZ, María Isabel. “Planificación Urbana y Regional para Santiago de Chile: Un aporte temprano para su sustentabilidad". Revista Digital Universitaria [en línea]. Julio de 2009 , vol. 10, $N^{\circ} 7$ [fecha de consulta: 14 de enero de 2013]. Disponible en: <http://www. revista.unam.mx/vol.10/num7/art46/ int46.htm>

RICOEUR, Paul. Del texto a la acción. Ensayos de hermeneútica II. México, Fondo de Cultura Económica, 2001.
PATAS DE PERRO IN THE CITY OF SANTIAGO DE CHILE IN 1960

Through the transparent presence of an adolescent portrayed in the novel, Patas de perro by Carlos Droguett ${ }^{1}$, we will attempt to decode the daily signs of social marginality staged in a sleepy, residential Santiago, a virtual space that has unified its violent negative to house Bobi, the cripple, whose experience of the urban landscape in 1960 has conditioned our perception of the slow and sustained urban degradation of the $21^{\text {st }}$ century.

But what is the history of the city that memory and the faltering steps of Bobi conserve while we move through the puddles, mud and blood of the Matadero neighborhood in this vicarious design of deformity and pain?

[...] I looked at the watery blood spilling over the sinks, I paused to look at the flayed horse head and later, later pieces of meat rained at my feet, they let me gather them, they demanded nothing more than a piece of meat and to eat it there, they asked nothing more, that was my contribution (Droguett, 1998, p. 94).

The articulation of that language builds the body-language of the imaginary world that envelops Bobi's monstrosity, while in that rupture constantly guarded by logic and legible history, our voice tries to dig through the ruined city walls to overcome the obscurity of the barbaric city. But what are the appropriate codes for (de) construct the symbolic places and the collective dreams in the historic memory of Santiago de Chile, in the tormented wandering of Bobi and over a city without arms, whose exclusion of the grotesque and deformed was organized and marked with specific territorial limits?

Bobi was from the Matadero-Franklin neighborhood, urban periphery of violence and death that bordered the pestilent waters of the Zanjón de la Aguadas, where tenement houses and ranches proclaimed the inconsistency of a state program for building working-class dwellings. "A purely working class neighborhood should be maintained and limited as such,"6 the real world affirms in the 1939 Brunner-Humeres plan for Santiago.

Thus, with the steps of Bobi and Carlos we intend to rebuild the space of the lower class inhabitants of Santiago de
Chile halfway through the 2oth century, a literary exercise that will allow us to redefine through diverse times, a past and a present to which we will give new meaning through a discursive turn. The text I have selected for semiotic study ${ }^{7}$ plays an active role in the ideological cultural mechanism of the mid-2oth century as it is the generator of a world model through its attempts to recover the active awareness of the social and historical man expressed within.

The novel, Patas de perro, partially recovers the memory and represents the remains of the city of Santiago updated at the beginning of the 21st century, whose memory and public spaces are being plundered and expropriated by (un) regulated architectural and landscape design introduced by the modern state and by the symbolic production of the Internet and television, paradoxes of integration that propose individualism and solitude surfing through diverse migratory fragments of virtual highways.

So we observe the inconsistency and delirium of a narrator who in his story attempts to deny the alleged unreality of Bobi's existence, an adolescent born in the iniquity of the Matadero neighborhood with beautiful dog feet. His words reveal a network of inconclusive clues, social phenomena associated with the history of Santiago de Chile between 1950 and 1964. This contextual situation allows us to speculate on the crowding of two million inhabitants in the capital, resulting from the peasant migration from the mines and fields, along with the malnutrition of $60 \%$ of the population of Santiago, considered the country's richest city between 1968 and 1969 (Collier y Sater, 1998).

In the story, Bobi's steps and gaze over the city and facades of the streets uncovers ghostly anachronistic subjects seeking a place to live and hide the tension of their fears in these disintegrated neighborhoods devoid of all compassion and emotional commitment.

[...] But I understand that others, spiritually deformed, dining room and kitchen people, plate and cup people must suffer, because you are not a deformed being, quite the contrary, because your form is new and whole and they are not. They are half-men, quarter-men... they are vermin, infected, domesticated beasts, voracious and calculating (Droguett, 1998, p. 65).

These words from Carlos, Bobi's protector and narrator of these tragic events, has been transformed in an autonomous act the reconstructs, in the different spaces of fiction, the acts of violence and segregation interiorized in the city since time immemorial. Then we learn from which exclusions and contradictions the discursive identities build in the same act of participation such as expressions of spatial memory and dialectic conglomerates of memories that constantly mobilize the reading of secret codes in out city.

Walter Benjamin tells us in his "Berlin Chronicle" (1950):

Anyone who seeks to approach his own past must become a man who digs [...] For the state of things is only storage, layers, that only after the most careful exploration will deliver the authentic values hidden within the earth (p. 212).

And Bobi's steps, generators of memories, evoke the map of his life in the old neighborhood of vice and misery, while the dig into a past where disturbing objects lay prisoners:

"I knew he would hit me. I was terrified of the brass buckle of his belt. I will never forget the smell of bronze between my lips when it split them" (Droguett, 1998, p. 170).

His father hated him, but he begged for alms to show him and had even bought a lantern, for the darkest places [...] (Droguett, 1998, p. 108).

Professor Bonilla picked up the knife from the table... with paternal care, he lifted his hand and slapped him and with other hand sliced him... Bobi felt the blood run down his cheek" (Droguett, 1998, p. 163).

Walter Benjamin affirms: "The encounter with the object liberates that past trapped within;" fragments of the past are then the bronze buckle, the knife and the lantern, everyday objects in the real world that transformed, in the interior of this violent world, into products of the excavation of disturbing possessions that have taken on their own form, weight and consistency to organize the temporal syntax that will materialize the real and concrete signs of the exile and tragic destiny of Bobi. The music is the whistle of the whip that forces him to beg for meat in the bloody stalls of the slaughterhouse; the ray of light is the lantern that illuminates his deformity as he devours repulsive pieces of meat; and the knife is the luminous skill of Professor Bonilla as he wounds him at prom night. These real objects, in the internal world of the story, make up virtual and temporary geometric coordinates to limit the territories from which Bobi is expelled to generate, with his steps, the framework of the social events that will give form to his history.

From alcoholic parents, monstrous, idiot children are born... others beget them and abandon them to the neighborhood, the city... it is an insult to have the boy in a school that has been open to receive human beings and not monsters spewed out by the infrastructure of the country (Droguett, 1998, p. 81).

The words of Bonilla expel Bobi, the perfect monster, from the lettered city to the cruel attacks of the pack. But from what institutions does he speak? We believe that in the discursive construction of the limited city of spawn, the violence and illegality of the territorial and social exclusion that has condemned the barbaric city at all times in our history are contained as symbols.

Then we need to recover the history of words in the subtle subversion of a multiple time to understand, in the 21st century, how the use of metaphors for perceiving the world of the excluded in a determined social caste has crystallized.

Thus we reiterate the autonomy of Carlos, the narrator whose delirium contradicts the hierarchies and exclusions he refers to, because the discursive identities that have generated his words are not pre-established in the symbolic dimension of an ideology. These dissident identities are built in the same discursive acts that allow them to freely participate in their own worlds of beliefs, and we propose to reflect on the identities in the text while we consider them as linguistic metaphors generating the spatial memory of the real city in the middle of the 2oth century. These dialectic conglomerates of memory represented in the language of literature allow us to invade and partially decode the dissident foci of the barbaric city in the spatial iniquity of an urban agglomeration that speaks to us of the social and 
economic structure of Chile in the fifties.

"When Bobi was born, his father was fired from the factory, his mother was at the point of death and he had to move, overwhelmed by the insults and embarrassment. At the grocer's they closed her account..." (Droguett, 1998, p. 93).

The institution has denied any link to the originality of Bobi's body, his equals fear losing their connection in the collective and mental space of the segregated city. However, Bobi's rebellion in his attempts to integrate into the originality of "a space of eyes full of age and submerged in wisdom, that no long look with hate, nor fury, nor solitude" (Droguett, 1998, p. 113) creates a scenario of an urban experience that brings us closer to an ideological discussion over the social production of spaces and places during the first half of the 2oth century.

\section{CARLOS AND BOBI, GENERATORS OF THE} SPATIAL MEMORY OF SANTIAGO IN 1960 Am I a human being?... I had the right to

ask this question... because until now life has been presented to me as a quality that does not correspond to me and the world as destination to which I have unlawfully arrived (Droguett, 1998, p. 111).

This question confirms our attempts to decipher the discursive identities of Carlos and Bobi while we consider their wanderings and dissident talk as generating dialects of the spatial memory of Santiago in the fifties. We know that the architectonic spaces are subjective territories that create real meanings for expressing the city's history and his materiality is conditioned by the cultural frameworks of those that design; thus the receptive and perceptive judgments and prejudices that affect their spaces and sociability, especially during the political scene in 1960, a utopian place where we begin to record the first urban and architectural concepts for integrating new social models and mobilizations whose central axis of social transformation is propelled by the Chilean state. ${ }^{8}$

Bobi's story is the urban experience of a deformed body that traverses the city in search of possible relationships that always fail, and the writing of Carlos is the map of a poetic mission illustrating the excluding sins of the collective body of our city.

How can we give form to the imbalance and discord of the social and urban body in Santiago de Chile? That is what the planners asked in the spatial reality of the " 60 s while the geometric coordinates of the spatial uprooting in the interior of the text speak to us:

Profesor Bonilla added that it was insolent to have the boy in a school that had been opened to receive human beings and not monsters vomited up from the country infrastructure [...] he inquired where were the legs of the boy named Bobi [...] that they had sent a dog and that this was not a school for dogs, nor a circus nor zoo, nor zoo, nor museum, nor exposition" (Droguett, 1998, p. 84).

The changes in the production of the space predicted transformations in the conceptual landscape to begin a new social order. This way, the conceptualization of "open spaces, green space, suburban area" that preserved the natural and cultural patrimony of Santiago de Chile, would correspond to the creation of a spatial utopia initiated from the State sphere with the proclamation in 1960 of the Intercity Plan for Urban Development (PRIS) $^{9}$. Some planners qualified the projects and urban renovations in 1960 as political strategies anticipating the appearance of the new man..$^{10}$

When Bobi finally leaves his home in the Matadero district, abandoned by the well-aimed relief of his parents, and Carlos was abandoned by the woman he was to marry, their steps and the timetable of their voices make up the image and form of a city inseparable from time and the memory contained in the sustainable development of the ' 60 s and its systems made up of green spaces destined for the amusement of population and the environmental healing strengthened by ecological corridors that try to united urban and rural areas (Pavez, 2009).

"Small house for couple in a quiet neighborhood, one block from Gran Avenida. Dogs not permitted" (Droguett, 1998, p. 72). The promise of paradise lost contained in the newspaper ad is the frenetic transition of Bobi and Carlos from is nightmarish streets toward the balance and beauty of the imagined city and to the vast deposit of a promising story contained in the health of its areas and avenues destined to achieve, in the past, the utopia of an institutionalism that regulated the protection of the natural landscape threatened by the urban expansion of the metropolis.

Looking for a place to live was the residue of the collective dreams of the citizens piled up over the territorial iniquity of the city in $1960^{11}$, a city whose totally new form could be the utopia of the design and protection of green spaces, rivers and ravines that can contain the perfect monstrosity of Bobi and the desire to forget of Carlos. Places designed for the perfection and happiness denied to them. A terrace and a patio full of flowers would be the architectonic construction of a place to live, the greenbelt utopia that announces and anticipates a new city in a quiet village, away from the hostility of the grand city that excludes Bobi's perfect monstrosity and would integrate him into this new production of urban space to apply for a different sociability, new men integrated into the plasticity of open forms, to feel, to breathe the air...

"We would go. We would look for a place in the foothills. We found a small house with a terrace and a patio full of flowers, a tiny kitchen, a clean, simple bathroom..." (Droguett, 1998, p. 88).

But houses, streets and cities are not only things and people. They are also thoughts, wills and participations that build up a context from which Bobi and Carlos are expelled with the imperative violence of a language: "The house is mine and I have the right to ask how you use it and why you use it like this!" (Droguett, 1998, p. 70); "My clients no- tify you that you must vacate the house on the 3oth of this month. Best regards, Gándara" (Droguett, 1998, p. 73). The end of the dreams in the infinite wickedness of Miss Estefanía, in her sluggish mouth remains the violent insertion of common sense to exclude from its spaces, the new and total forms that represent the beautiful dog feet of Bobi: "We'll move. Right? Yes, Bobi, we will move, we will find a nice little house" (Droguett, 1998, p. 81).

We will enter the house, that looked brighter and somehow bigger now... Puente Alto... we felt the dry air full of echoes coming down the sunny mountain, when I asked if he would like to move to this town, provincial, quiet, grounded to the earth [...] between the tall mountains along gentle rivers, he stared at me full of joy and told me that he had always been surprised by my desire to stay in Santiago, when I had nothing to do in that huge, hostile city [...] (Droguett, 1998, p. 111).

Will Puente Alto be the city project imagined by Bobi and its new forms in the fictitious framework of a new spatial legality? Would it be the salvific utopia that would rescue him from the crowding, stench and pestilence of the Matadero neighborhood and from the tragic institutional persecution embodied by his father, school and the police?

Bobi, I told him, the truth is the people of this village have no eyes, or their eyes are like those of fish, polished by the sea, eyes without edges that feel no pain, eyes full of age submerged in wisdom that no longer shoot glances of hate, nor fury, nor loneliness (Droguett, 1998, p. 113).

And it will only be the looks "full of age submerged in wisdom" in these transparent spaces that will fix on an interchange of subjectivities, the unformulated acceptance of Bobi. And so, Puente Alto will be the last bastion of daydreams to be offered to a dying Bobi, stalked by the institutionalism that exercises violent prevention against abnormality.

\section{BOBI, FAWNS, SATYRS}

\section{AND SACRED SPACE}

We unearth the extraordinary luminosity of the Dionysian myth ${ }^{12}$, from the school dance Bobi attends, in the sweet sensuality that exercises the perfect shapes of his dog feet in the comparison that joins it to its mal-formed kin, the satyrs, fawns and centaurs. In the negative of this gallantry to accept the order and horror imposed by the titans, the acceptance of the divine ecstasy remains latent in the lethargic dance that updates and performs Bobi to attempt the redemption that would reunite him with the human world. From this perspective, our reading is converted to an exercise of the imagination that will manage effective maps for containing, from language and memory, impossible spaces whose transformation in pockets of reverie makes possible this utopia of the new sociability and the idyllic spaces that could contain it. ${ }^{13}$

I danced, Bobi told me, I really danced with Silvia, Enriqueta, Matilda, we danced a lot, we were hot in the face, the sun on our lips, the music moved our feet. I took their waists. They turned pale and thoughtful.

Silvia told me I danced very well, asked why I never went out at night, told me that at night the dances shine at the end of the street. He whispered, pale, happy and forgotten, he began to whistle softly (Droguett, 1998, p. 159).

But what is the meaning of the Dionysian world in the steps that Bobi dances moving him to demarcate his sacred space in the fragmented city? We must then speak of his wisdom and insight in the search for lost unity and the affirmation of live above the pain of rejection and abandonment beyond that of superficial optimism.

Friedrich Nietzsche said:

Life is indestructibly powerful and pleasurable, that comfort appears with corporeal evidence, like a chorus of satyrs, like a chorus of natural beings, that live, so to speak, inextinguishable behind all civilization and, despite the passing of generations and history, remains eternally the same (Nietzsche, 1973, p. 77).

However, we understand that there is no place for orgiastic dancing in the forest and the fantasy of a magic path to establishing this lost unity has been broken by the strident actions of professor Bonilla and the terrifying efficiency of the political and family titans; Bobi, hurt and humiliated, is obliged to leave the school dance after his triumph and the fervent applause from his audience.

This knife... the teacher said and smiled now with fear, with real fear, will commence the Kermesse, do not be afraid, be brave, look how brave Bobi is and he turned towards him, putting the knife between his teeth and as he began to yell, he threw himself down on the steps and screamed furiously, but the people did not jump up nor flee, the people were not afraid. They were applauding [...] (Droguett, 1998, p. 163).

The audience applauded earnestly and its nymphs, blinded by the dance ritual, joined Bobi, the lost son, on the magical path of unity. The forgetting and drunkenness confer Bobi the triumph and the exultation of his dog ways, while the maenads of gallantry participate in the frenzy of his divine transfiguration in this magic space, the abyss that marks his separation from "the others." "When he felt that they called him and heard their applause, he stood up, put on his mask and walked away (...) (Droguett, 1998, p. 161).

We wonder over the meaning of the violence of professor Bonilla toward Bobi's dance with his classmates? Then we return to the Bacchic myth, to Euripides, $\mathrm{V}$ century B.C., to these stanzas of The Bacchae ${ }^{14}$ :

I have heard of new evils in the city that 
son for the oppression of the state against the excesses of nature (Euripides, 2005).

Bobi looked at the patio full of lights and people and felt intimidated, admired that five minutes before it had been him dancing gently around a nice, perfumed, vaporous creature, raising his hands with energy to the rhythm of the dance, moving his masked and fevered face towards the mouth of the inquirer (Droguett, 1998, p. 169).

Bonilla, dark pedagogue with worn clothes, frightened of the seduction exercised by the breathless dances of passion for the triumph of existence and the exuberant feeling of transfiguration -just like Pentheus, king of Thebes-feels bound to destroy this excess and oppose the reason:

The teacher snatched the mask and rose to the applause [...] raised his hand and slapped him and with the other he thrust a knife and the mask jumped like a spring cap and Bobifelt blood run down his cheeks [...] If the music stops, he thought, they will kill me, and he looked at the professor's face watching him like a beast, a murderer, a depraved monster [...] (Droguett, 1998, p. 165).

The mask and the music mark the transformation and the hero's sacrifice, his body tortured and chained lying in a dirty police station; the excess placated by reason represents the end of a unifying path to integrating with nature, in these minor gaps in the urban landscape one can see the icy breeze, the rivers, the hollows, the ravines ${ }^{15}$. Bobi would represent the last hope for redemption in a universe fenced in by titans "you are the mysterious carrier of a revolutionary form of human being, much more noble, more loyal [...] if Nature has changed in your body, it has brought you as its emissary, like a very special spy" (Droguett, 1998, p. 248).

After fleeing the police station, Bobi walks along the San Cristobal Hill, the banks of the Mapocho River and the Quinta Normal Park in Santiago. On these trajectories it is impossible to hide nor escape because the image and the form of our city is also our body, inseparable from time and memory "a boat with an enamored couple in the middle of the water, the sleepy or resigned ice cream vendor, the wandering photographer, downright angry and barbaric".

These are our diluted visions, faded postcards converted into our poetic mission to recover in Bobi's pain, time, memory and the images of our destroyed city.

Then Bobi's adventure has not only been a matter of bodies, a lone body that leaves itself and exposes itself to others in the exultation of a particular beauty: his flight becomes a prescient prediction of a new city marked by banality and meaninglessness:
You look at a thin and transparent world, huge tree of paper, filthy, false water, an excellent imitation, water of crumpled paper paper men and women, paper smiles, paper words that fly away with the coal smoke from the train line, I was never so removed from life as in that afternoon in the Quinta, but it was nice because the dogs walked alongside me [...](Droguett, 1998, p. 201).

We wonder along with Bobi how removed we will be from real life in the current simulation of a new electrified sociability? Why was Puente Alto the end of our journey where "they don't look at you as if you were the monster that they are? (Droguett, 1998, p. 82). But we understand that this nostalgia was a trap and that in these sacred spaces, salvation is no longer possible, the present has been a conviction, eternal hopeless lucidity. Puente Alto was transformed at the beginning of the 21st century in places of social conflict, alien and hostile to the interpretative codes of urban policies that proposed the green space utopia of 1960. A utopia that ended in 1979 with the pressure of the real estate development sector that pushed for the release of the land markets ${ }^{16}$ and eliminated the regulations on urban limits. That situation produced a serious residential segregation, especially in Puente Alto (a recipient selected for the policies of eradication and location of slums and low-income housing together with the highest rates of criminal activity and crowding) together with controversy generated by the 2012 CASEN census findings of 629,861 inhabitants of which 100,000 people live in poverty.

So Bobi joining this pack is our consolation; Friedrich Nietzsche said:

That consolation appears with bodily evidence, like a chorus of satyrs, like a chorus of natural beings, that live, so to speak, inextinguishable behind all civilization and which, despite the coming and going of generations and history, they remain eternally the same" (Nietzsche, 1973, p. 77).

We take away the apparent simulation from Bobi and his eternity of an object that emerges almost hidden among so many words. The flute and the melancholy notes of the Mozart's clarinet concerto, the flute distractedly close to the radio receiver, the flute apparently forgotten and with wrinkled clothes, the flute now wielded triumphantly by Bobi, when his footsteps mark the moist earth that leads him to the pack.

The flute, no. The flute was not there, I went to the patio, I went into the dark street, I looked at the earth and there where his tracks, I bent down to look at them... My God! I yelled with fury as the tears burst from my eyes (Droguett, 1998, p. 31). ARQ
"Patas de perro" could be translated as "dog feet". Editorial Zig-Zag published the first edition of this novel in Santiago de Chile in 1965.

"Matadero" is the Spanish word for slaughterhous (Trans. note).

3 All quotes from the novel Patas de perro correspond to the first issue published by Pehuén Editores in September 1998 within its "Premios Nacionales de Literatura" Series.

4 The "house for the poor" issue stopped being only a charity matter related to the Catholic Church and the religious people at the Conservative Party since the enactment of the 1906 Workers' Housing Act and the creation of a Superior Council of Workers' Housing. In the mid 2oth century, the Chilean State became an active agent in a process that led in 1953 to the Decree in Force of Law No 224, that generated later the Chilean General Law for Buildings and Urbanization in order to regulate a scientific development of urban growth. This idea was inspired by new and attractive international urban experiences, such as the Greater London Plan by Patrick Abercrombie (1944) and the utopian city of Brasilia, created by Oscar Niemeyer (1957). This explains the 1960 "Plan Intercomunal para Santiago", which replaced the previous urban plan by Brunner and Humeres.

5 The "Zanjón de la Aguada" is a natural watercourse that runs from East to West through the Southern districts of Santiago. Historically its streambed has been used as wasteland and open sewage; during the second half of the 2oth century its banks hosted the first industrial shantytowns in Santiago. The area is currently undergoing major renewal operations (Ed. note)

6 Armando de Ramón (2000) stated that the Brunner-Humeres urban plan supported a spatial segregation based on social classes. According to the document, "the streets and boulevards where luxury residences or chalets surrounded by gardens stand" shouldn't be close to lower-class housing because they could affect negatively the value of the properties. Brunner's skepticism was rooted in the alleged "ignorance of ordinary people": facing the failure of the so called "Law on cheap housing" and due to the lack of resources, these men would end up building "a shack made out of boxes, timber scraps and discarded cans".

7 Iuri Lotman demonstrated in his article On semiosphere (1984) that semiotics is a discipline capable of dealing with the study of complex social life and the relationships between the world and the human being. Regarding textual semiotics, its most distinctive element is the global thesis on the functions of the literary text as cultural sign. Our research focuses on textual cultural signs that -in literature-represent a particular enunciative production and reception orientated to grasp and integrate the understanding of the real world (Favi, 2012)

8 In the article Bicentenario: remodelación urbana. Utopías y anticipaciones para el hombre nuevo, the author Alfonso Raposo Moyano reflects on the notion of "remodeling" and the CORMU (Corporación del Mejoramiento Urbano or Urban Improvement Corporation, created in 1965) playing the role of one of the executive offices that, during the ' 60 s, would anticipate the spaces to mark the territories that the new man would build. In this context, production of public space is considered as a social laboratory coming from the public realm created by the political and administrative bureau of the Chilean Christian Democratic government.

9 The "Plan Intercomunal de Desarrollo Urbano pris" (1960) proposed, among other points: I) To reforest low-quality agricultural land where consumption ravaged the woods; II) To implement a "constellated cities" plan, this is, a group of interconnected urban settlements of different size and luminosity; III) Raise the number of leisure areas in valleys, lakes, lagoons and high mountains. Chile, Public Works Office.

10 Both the 1960 pris -that contained certain regional and urban programs for Santiago de Chile- and the 1964 Micro-Regional plan for the Santiago basin -that included the idea of natural landscape regulation in the periphery threatened by urban sprawl- tried to strengthen ecological corridors within central Santiago. Quoting one of the pris sections: "The use of suburban land is regulated in order to promote and boost agricultural production, creating at the same time a greenbelt between urban and suburban areas". These projects are evidence of the relevant experience that the Chilean State, as a planner, implemented to rationalize urbanization, energy and public transportation services. It aimed to human settlements designed to integrate urban form and nature.

11 Due to population growth and the arrival of a arge number of rural immigrants, during the " 60 s there were impressive social movements related to housing. The State reacted, enacting the Law No 16.391 that in 1965 created the Housing and Urbanism Office (MINVU), whose mission was to intensify the urban planning efforts. The Housing Corporation (CORVI), originally conceived as a provider of temporary residencies for the people affected by rainstorms, offered intermediate solutions to address the housing crisis

12 "Under the magic of the Dionysian, not only does the bond between man and man lock itself in place once more, but also nature itself, now matter how alienated, hostile, or subjugated, rejoices again in her festival of reconciliation with her prodigal son, man. The earth freely offers up her gifts, and the beasts of prey from the rocks and the desert approach in peace [...] Now is the slave a free man, now all the stiff, hostile barriers break apart, those things which necessity and arbitrary power or "saucy fashion" have established between men: the slave becomes a free man, the noble and the humble peasant get together and join the same Bacchic chorus". The Birth of Tragedy (Nietzsche, 1973, p. 232). 13 Nymphs, satyrs and centaurs were part of the Bacchic procession; their lethargic dances in the woods traced a magic path that separated them from the rest of humankind but because of the roar of the dancers they, -natural beings- subjugate and reconcile humans and nature. Mother and prodiga son reconcile, the earth offers up her gifts and the beasts of prey approach in peace.

4 Dionysian nymphs are Bacchu's servants an playmates. Euripides dedicated one of his tragedies, The Bachaee, to these crazy, reckless beauties; it was staged in 405 b.C. (Daudi, 1965).

15 Cf: Metamorphoses, Book III. Publius Ovidius Naso (43 b.C.) "Pentheus shouts: What madness has stupefied your minds, children of the serpent, people of Mars? Can the clash of brazen cymbals, pipes of curved horn, and magical tricks be so powerful that men, who were not terrified by drawn swords or blaring trumpets or ranks of sharp spears, are overcome by the shrieks of women, men mad with wine, crowds of obscenities, and empty drumming?" (p. 43).

16 According to the MINVU: "The use of land must be regulated through flexible rules; the demands of market should define those rules". On the other hand, "procedures will be defined and restrictions will be removed in order to allow natural growth of urban areas according to the market trends".
GLORIA FAVI | Spanish Professor, 1971; Master in Literature, 1990 y (c) Doctorate in Literature, Universidad de Chile. Reasearcher in the Deparment of Research and Development of the Universidad de Chile since 2007 and beginning in 2011 formed part of the editorial committee of the indexed journal Cifra Nueva, edited by the Universidad de Los Andes en Trujillo, Venezuela. She wrote the book Chronicles of Poverty, edited 